\title{
Artificial neural network, genetic algorithm, and logistic regression applications for predicting renal colic in emergency settings
}

\author{
Cenker Eken • Ugur Bilge • Mutlu Kartal • Oktay Eray
}

Received: 3 March 2009 / Accepted: 13 April 2009 / Published online: 3 June 2009

(C) Springer-Verlag London Ltd 2009

\begin{abstract}
Background Logistic regression is the most common statistical model for processing multivariate data in the medical literature. Artificial intelligence models like an artificial neural network (ANN) and genetic algorithm (GA) may also be useful to interpret medical data.

Aims The purpose of this study was to perform artificial intelligence models on a medical data sheet and compare to logistic regression.

Methods ANN, GA, and logistic regression analysis were carried out on a data sheet of a previously published article regarding patients presenting to an emergency department with flank pain suspicious for renal colic.

Results The study population was composed of 227 patients: 176 patients had a diagnosis of urinary stone, while 51 ultimately had no calculus. The GA found two decision rules in predicting urinary stones. Rule 1 consisted of being male, pain not spreading to back, and no fever. In rule 2 , pelvicaliceal dilatation on bedside ultrasonography replaced no fever. ANN, GA rule 1, GA
\end{abstract}

The views expressed in this paper are those of the author(s) and not those of the editors, editorial board or publisher.

C. Eken $(\bowtie) \cdot$ M. Kartal $\cdot$ O. Eray

Department of Emergency Medicine, Akdeniz University Medical Faculty,

Dumlupinar Bulvari, Kampus,

07059 Antalya, Turkey

e-mail: cenkereken@akdeniz.edu.tr

M. Kartal

e-mail: enmkartal@yahoo.com

U. Bilge

Department of Biostatistics, Akdeniz University Medical Faculty, 07059 Antalya, Turkey

e-mail: ubilge@akdeniz.edu.tr rule 2, and logistic regression had a sensitivity of 94.9, $67.6,56.8$, and $95.5 \%$, a specificity of $78.4,76.47,86.3$, and $47.1 \%$, a positive likelihood ratio of $4.4,2.9,4.1$, and 1.8 , and a negative likelihood ratio of $0.06,0.42,0.5$, and 0.09 , respectively. The area under the curve was found to be $0.867,0.720,0.715$, and 0.713 for all applications, respectively.

Conclusion Data mining techniques such as ANN and GA can be used for predicting renal colic in emergency settings and to constitute clinical decision rules. They may be an alternative to conventional multivariate analysis applications used in biostatistics.

Keywords Artificial neural network - Genetic algorithm . Logistic regression · Renal colic · Emergency department

\section{Introduction}

There are many articles in the medical literature measuring diagnostic tools for patients with acute flank pain. The main purpose of these studies is to find the safest and most costeffective way to detect urinary stones in the emergency department (ED). Logistic regression is mostly used in these studies in processing multivariate data. Alternative methods in data mining, also known as artificial intelligence, should also be used to evaluate the medical data sheet.

An artificial neural network (ANN) is an information processing tool that is inspired by the structure and function of the human brain. The human central nervous system is composed of a series of interconnecting neurons separated by synapses, and scientists have demonstrated information transfer via a series of action potentials [1]. The brain learns by adjusting the number and strength of these connections. 
McCulloch and Pitts [2] first described ANNs as a method of information processing using a network of binary decision elements or "neurons." Later efforts were made in order to explain complex processes of the central nervous system [3]. A neural network is composed of a series of interconnecting parallel nonlinear processing elements (nodes) resembling biological neurons.

A genetic algorithm (GA) $[4,5]$ is a good method for finding quick solutions to hard combinatorial optimization problems where there are too many combinations to search and were first put forward by John Holland as a search and optimization technique. A GA typically generates a random population of possible rule hypotheses and tests them with a given fitness function. In a standard GA, solution hypotheses are represented as code strings; this is also called a genotype where genetic operators such as crossover and mutation are applied. The resulting phenotypes are then assessed by applying the fitness function to each one of them. At each iteration the GA applies the principles of natural evolution to the population of hypotheses, that is, selecting the fittest hypotheses using the fitness function as well as applying mutation and crossover to the population.

In our first article [6], we tested a current algorithm and investigated the clinical characteristics of renal colic patients in the ED. The objective of this study was to compare predictive values of multiple statistical models for diagnosing renal colic patients in the same data set.

\section{Material and methods}

Data collection

The data sheet of our previously published article [6] on renal colic was used to perform ANN, GA, and logistic regression analyses. We additionally included the body temperature of patients into the analyses although it had not been included in the previous data sheet. The data set consists of the following parameters:

\begin{tabular}{ll}
\hline $\begin{array}{l}\text { Parameter } \\
\text { Gender }\end{array}$ & $\begin{array}{l}\text { Type } \\
\text { Coded }(2 \text { codes, i.e., male, female })\end{array}$ \\
Number of attacks & $\begin{array}{l}\text { Coded }(2 \text { codes, i.e., first, more than one }) \\
\text { Coded as discrete variable for GA }\end{array}$ \\
Triggered by exercise & Coded $(2$ codes; 1 for yes and 0$)$ \\
Previous presentation & Coded $(2$ codes; 1 for yes and 0$)$ \\
History of urolithiasis & Coded $(2$ codes; 1 for yes and 0$)$ \\
Family history of & Coded $(2$ codes; 1 for yes and 0$)$ \\
urolithiasis & Coded $(2$ codes; 1 for yes and 0$)$ \\
Nausea & Coded $(2$ codes; 1 for yes and 0$)$
\end{tabular}

\begin{tabular}{|c|c|}
\hline Parameter & Type \\
\hline Vomiting & Coded ( 2 codes; 1 for yes and 0 ) \\
\hline Sweating & Coded ( 2 codes; 1 for yes and 0 ) \\
\hline Fever & Coded ( 2 codes; 1 for yes and 0 ) \\
\hline Dysuria & Coded ( 2 codes; 1 for yes and 0 ) \\
\hline Hematuria & Coded ( 2 codes; 1 for yes and 0 ) \\
\hline Unable to void & Coded ( 2 codes; 1 for yes and 0 ) \\
\hline $\begin{array}{l}\text { Tenderness in } \\
\text { costovertebral region }\end{array}$ & Coded ( 2 codes; 1 for yes and 0$)$ \\
\hline $\begin{array}{l}\text { Tenderness on ureteral } \\
\text { tract }\end{array}$ & Coded ( 2 codes; 1 for yes and 0 ) \\
\hline Suprapubic tenderness & Coded ( 2 codes; 1 for yes and 0 ) \\
\hline Abdominal rigidity & Coded ( 2 codes; 1 for yes and 0 ) \\
\hline Rebound & Coded ( 2 codes; 1 for yes and 0 ) \\
\hline Positional discomfort & Coded ( 2 codes; 1 for yes and 0 ) \\
\hline $\begin{array}{l}\text { Positive urine analysis } \\
(\geq 10 \text { red blood cells) }\end{array}$ & Coded ( 2 codes; 1 for yes and 0 ) \\
\hline $\begin{array}{l}\text { Pelvicaliceal dilatation } \\
\text { on bedside } \\
\text { ultrasonography }\end{array}$ & Coded ( 2 codes; 1 for yes and 0 ) \\
\hline Colic pain & Coded ( 2 codes; 1 for yes and 0 ) \\
\hline \multirow[t]{5}{*}{ Radiation to the groin } & Coded ( 2 codes; 1 for yes and 0 ) \\
\hline & $0=$ no radiation \\
\hline & $1=$ radiation to the belly \\
\hline & $2=$ radiation to the groin \\
\hline & $3=$ radiation to the back for GA codes \\
\hline
\end{tabular}

Statistical analyses

\section{Artificial neural network}

A feed-forward ANN with back-propagation was performed by JMP (release 6.0, a business unit of SAS). In large data sets, a data set is divided as a training set and a test set to avoid overfitting which is a problem when ANN learns the training set too accurately yet it cannot generalize when presented with a new test set. However, for the small data sets, the k-fold cross-validation model is suggested to avoid overfitting. The k-fold cross-validation method separates the data into $\mathrm{k}$ sets and assigns one of the $\mathrm{k}$ sets as the test set and the remaining as the training set. We performed 10-fold cross-validation with 2 hidden units, 500 iterations, and 20 tours. The overfit penalty was assigned as 0.001 and convergence criterion was chosen as 0.00001 .

\section{Genetic algorithm}

The GA program SimMine is part of a data mining software package developed by SimWorld Limited, UK (www.simworld.co.uk). The system allows the selection of 
flat or hierarchical chromosome types and allows preprocessing the data by scaling, taking first differences and dividing continuous data into categories. As GA normally operate on categorical data, when the input parameters are a mixture of numerical and coded values, the system converts numerical values into a number of categories. The system allows users to select the total number of chromosomes (the population size) and the number of top scoring individuals that survive to the next generation.

\section{Fitness score}

When the GA puts forward a rule hypothesis, the system counts the number of samples that are covered by this hypothesis. By this we mean positive_count; for example, if we are trying to find a rule that explains all the patients that have urinary stones, then positive_count would be the total number of patients with urinary stones. Then the system tests each hypothesis by counting the number of patients that are covered by the hypothesis, and this is the true_positive. Similar logic is applied to find negative_count, which is the total number of samples not covered by the hypothesis (i.e., total samples minus positive_count), and the false_positive, which is the total number of samples the hypothesis would falsely find positive, which is undesirable. So the fitness function would be:

Fitness score $=($ true_positive/positive_count $)$

$$
\text { - (false positive/negative_count) }
$$

As can be seen above, the GA could achieve a maximum score of 1 when it identifies all positives correctly, and would have a false_positive of 0 .

We used the software previously on data mining tasks at Akdeniz University Hospital with good results [3].

\section{Logistic regression}

The Statistical Package for the Social Sciences (SPSS 13.0 for Windows) was used for binary logistic regression analysis. Like in GA and ANN, 24 independent variables were assigned to predict the renal colic as the dependent variable. A stepwise forward logistic regression analysis was performed. We tested the fitness of the logistic regression model with the Hosmer-Lemeshow goodnessof-fit statistic.

To compare the overall performances of all applications, sensitivity, specificity, positive predictive value, and negative predictive value of all applications were determined. And also a receiver-operating characteristic curve (ROC) analysis was performed; $p \geq 0.05$ was accepted as significant.

\section{Results}

The study population was composed of 227 patients; 147 (64.8\%) patients were men and their mean age was $38.4 \pm$ 14. Diagnostic studies and follow-up revealed 176 patients with urinary stone, while 51 had no calculus. Demographics of 24 independent variables are shown in Table 1.

\section{Artificial neural network}

ANN revealed 174 of 176 patients with renal colic and 40 of 51 patients without renal colic. It assigned $11(21.6 \%)$ patients falsely as having renal colic and 9 (5.1\%) renal colic patients as having no calculus. So it had a sensitivity of $94.9 \%$ and a specificity of $78.4 \%$. The positive predictive value of ANN was $93.8 \%$ and negative predictive value was $81.6 \%$. The positive likelihood ratio (PLR) of ANN was 4.4 and negative likelihood ratio (NLR) was 0.06. The area under the curve (AUC) for the ANN model was $0.928(p=0.000)$. Figure 1 shows an ANN diagram with three layers: input layer, hidden layer with two nodes, and output layer.

\section{Genetic algorithm results}

We used the GA population size of 128 , and the top scoring 32 individuals were selected as fit to survive in the next generation of solutions; the search was stopped after no improvement was seen for the last 25 iterations of each run. The default value for the crossover rate was $60 \%$, and the mutation rate was $20 \%$ per chromosome.

When we apply the GA, the system quickly converges into simple rules. Modifying the crossover rate or the mutation rate does not improve the results.

The best rule to explain urinary stone $=\mathbf{1}$ is presented below:

Rule 1:

Kidney stone (1): gender (1) AND spread (0 OR 1 OR 2) AND no fever (0)

Total samples $=227$

Positive_count $=176$ (total of 176 cases of urinary stone is 1)

True positive $=119$ (the rule above explains 119 of the 176)

Negative_count $=51$ (total of 51 cases where urinary stone is 0)

False positive $=12$ (the rule is wrong in 12 of 51 cases)

Fitness score $=0.4408$

Sensitivity $=67.61 \%$

Specificity $=76.47 \%$ 
Table 1 Demographics and univariate data analysis of patients with renal colic

US ultrasonography

Positive predictive value $=90.84 \%$

Negative predictive value $=40.6 \%$

Positive likelihood ratio: 2.9

Negative likelihood ratio: 0.42

Area under the curve $=72.04 \%$

This rule can be explained in a more articulate form as "the patient has urinary stone if: the gender is male, and the pain radiates to groin and belly or no pain radiation (codes: 0,1 , or 2), and has no fever". As there are 4 types of pain (they are $0,1,2$, and 3 as can be seen in the "Data collection" section), we can reword the rule as "the patient has urinary stone if: the gender is male, and the pain does not radiate to the back, and the patient has no fever."

A second rule that was also found, which has slightly less fitness value but worth reporting, is shown below:

\section{Rule 2:}

Urinary stone (1): gender (1) AND pain radiation (0 OR 1 OR 2) AND pelvicaliceal dilatation on bedside ultrasonography (1)

Total samples $=227$

Positive_count $=176$ (total of 176 cases of urinary stone is 1)
True_positive $=100$ (the rule above explains 100 of the 176)

Negative_count $=51$ (total of 51 cases where urinary stone is 0 )

False positive $=7$ (the rule is wrong in 7 of 51 cases)

Fitness score $=0.4309$

Sensitivity $=56.82 \%$

Specificity $=86.27 \%$

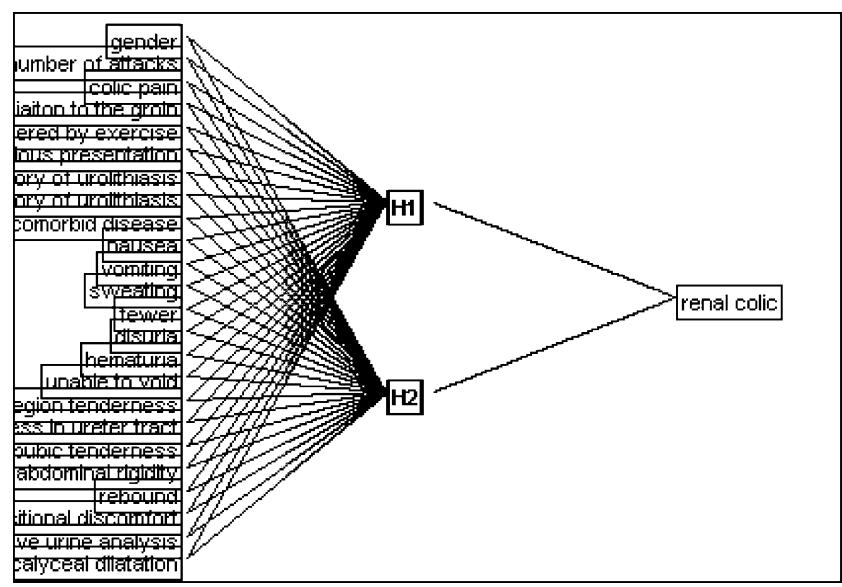

Fig. 1 ANN diagram exhibiting the input layer with 24 nodes, hidden layer with 2 nodes, and output layer 
Positive predictive value $=93.46 \%$

Negative predictive value $=36.7 \%$

Positive likelihood ratio: 4.1

Negative likelihood ratio: 0.5

Area under the curve $=71.55 \%$

This rule can be also explained in a more articulate form as "the patient has urinary stone if: the gender is male, and the pain does not radiate to the back, and has pelvicaliceal dilation on bedside ultrasonography (US)."

\section{Logistic regression}

With the forward stepwise conditional method, the logistic regression model with 24 independent variables mentioned above revealed 168 of 176 patients with renal colic and 24 of 51 patients without renal colic. So it had a sensitivity of $95.5 \%$ and specificity of $47.1 \%$. The positive predictive value was $86.2 \%$ and negative predictive value was $75.3 \%$. The positive predictive value of logistic regression was 1.8 and negative predictive value was 0.09 . The AUC for the logistic regression model was $0.841(p=0.000)$.

Gender (to be male) [odds ratio (OR): 5.567, 95\% confidence interval (CI): 2.34-13.24, $p=0.000]$, history of urolithiasis (OR: 2.72, 95\% CI: 1-7.3, $p=0.047$ ), and nausea (OR: 3, 95\% CI: $1.12-8.5, p=0.029$ ) were found to be independent factors in predicting urinary stones in logistic regression analysis.

Table 2 shows the overall performances of each model. Figure 2 depicts the comparison of ANN and logistic regression applications after the ROC analysis according to the prediction rates. The AUC for GA rules may be realized as smaller than ANN and logistic regression. However, these findings represent the binary outcomes of GA rules. Also, a ROC analysis was performed to see the overall performances of each model after the outcomes were dichotomized to have renal colic or not. Figure 3 shows a comparison of the three models with ROC analysis.

\section{Discussion}

We are in the process of applying the technology to other data mining tasks in the hospital databases. We are aiming for an automated approach to explore and reveal hidden, unknown relationships in data. We wanted to enrich the ANN and GA data mining techniques with a logistic regression processing module and also to compare the fitness functions using statistical techniques.

In medical research, efforts are expended to detect a disease most accurately or predict the prognosis of a specific group of patients. Logistic regression analysis is the most frequently used statistical analysis for evaluating multiple independent variables through a dependent outcome in medical research. Although ANN and GA have been used commonly in other industries, they do not have widespread use in medicine so far. Here we show that both ANN and GA can be used for constituting prediction models and decision rules as well as logistic regression analysis.

ANN emerged as the best technique in predicting renal colic according to the results of this study. For ANN both the sensitivity (94.9\%) and PLR (4.4) were higher than for the other models. The AUC was also found to be higher than with logistic regression and GA. However, overfitting is a major problem in ANN applications. In order to prevent overfitting, the data set must be separated randomly as the training set and test set. After the learning process of ANN by using the training set, the model should be tested in unseen data, a test set and sometimes a validation set. However, this is not always possible for small data sets. The k-fold cross-validation that we performed in this study separates the data sets into $\mathrm{k}$ groups and uses the groups as the data test set and the remaining as the training set. Although k-fold cross-validation was used, to test ANN in an unseen data test would be better. Even so the results are promising for ANN. ANN previously has been used for predicting genetic factors in stone disease [7], stone composition [8], spontaneous passage of ureteral calculi [9], and outcome after extracorporeal shock wave lithotripsy (ESWL) [10]. So far there is only one study detecting the performance of ANN in predicting the presence of renal calculi [11]. Tanthanuch and Tanthanuch studied 168 patients with renal calculi, 100 patients for the training set and 68 patients for the test set. After assuming a probability of $65 \%$ or more as having renal calculi, they found that ANN was $100 \%$ accurate in predicting renal calculi for the testing data. However, the study population they investigated
Table 2 Overall performances of each application

\begin{tabular}{llllll}
\hline Application & Sensitivity & Specificity & PLR & NLR & AUC \\
\hline ANN & 94.9 & 78.4 & 4.4 & 0.06 & 0.867 \\
GA rule 1 & 67.6 & 76.47 & 2.9 & 0.42 & 0.720 \\
GA rule 2 & 56.8 & 86.3 & 4.1 & 0.5 & 0.715 \\
Logistic regression & 95.5 & 47.1 & 1.8 & 0.09 & 0.713 \\
\hline
\end{tabular}




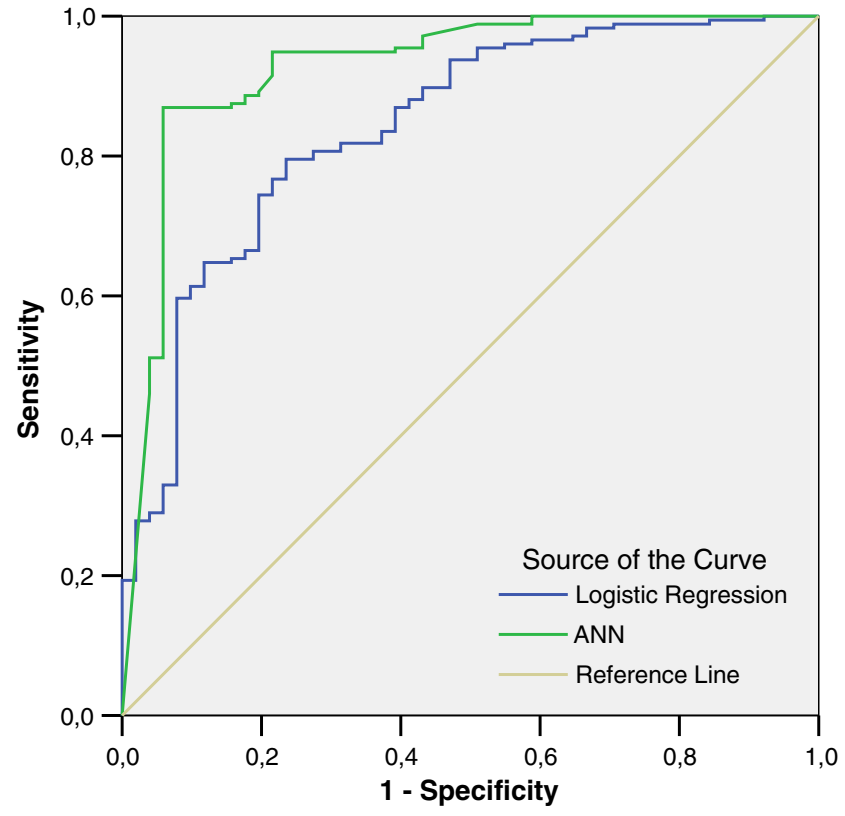

Fig. 2 ROC analysis of logistic regression and ANN in predicting the probability of urinary stones

consisted of only patients with renal calculi. Thus, ANN was trained and tested only in patients with renal calculi. The performance of ANN was not tested in patients without a renal calculus. This is a limitation of their study.

GAs are relatively new search techniques which have been used in optimization, scheduling, and planning tasks, and there is a huge amount of background literature on them. As in other industries, they are increasingly being used in medical data mining tasks. Our application of GA found useful relationships for predicting renal colic. Especially the PLR of the second rule was worth mentioning. The advantage of GA is to discover simple predictive rules. In the first rule of GA, to be male, having pain not radiating to the back, and having no fever composed the independent variables. In the second rule, pelvicaliceal dilatation on bedside US replaced having no fever. The PLR (4.1) of the second rule seems better than the first rule. And the specificity of the second rule (86.3\%) was better than the first rule $(76.5 \%)$. However, the sensitivity of the first rule $(67.6 \%)$ was better than the second rule $(56.8 \%)$.

Logistic regression emerges as the best model in this study in excluding renal colic. The NLR of logistic regression was 0.09 , and also the NLR of ANN was 0.06 , which is also reasonable to mention. But the overfitting problem has to be taken into consideration for ANN as mentioned above. Furthermore, gender (to be male), nausea, and history of urolithiasis were found to be the independent variables in predicting urinary stones.

One can also constitute simple prediction rules both by ANN and logistic regression applications. After analyzing the significant variables in the univariate analysis or by using the likelihood ratios, it is possible to compose a prediction model by assigning significant variables into the ANN and logistic regression analysis. Furthermore, the independent variables that are statistically significant in a multivariate regression analysis can also be used to compose a prediction model. We preferred to use all of the variables ( 24 variables) for all the models in order to prevent bias. Different models for renal colic need to be tested in further studies.

\section{Conclusion and future work}

Data mining techniques, particularly ANN rather than GA, have been used in order to solve problems in the ED like diagnosing pathological conditions such as cardiac ischemia, craniocervical junction injury in trauma patients, and pneumonia, predicting the prognosis of patients in the ED, and also possible ED visits of patients because of respiratory symptoms [12-16]. This study also showed that data mining techniques such as ANN and GA could be used for predicting or excluding renal colic in emergency settings and to constitute clinical decision rules. Data mining techniques should be useful tools to solve the sophisticated issues in the ED in the future. They may also be an alternative to conventional multivariate analysis applications used in biostatistics. Further studies are needed to validate these data mining techniques in predicting either renal colic or complicated points in the ED.

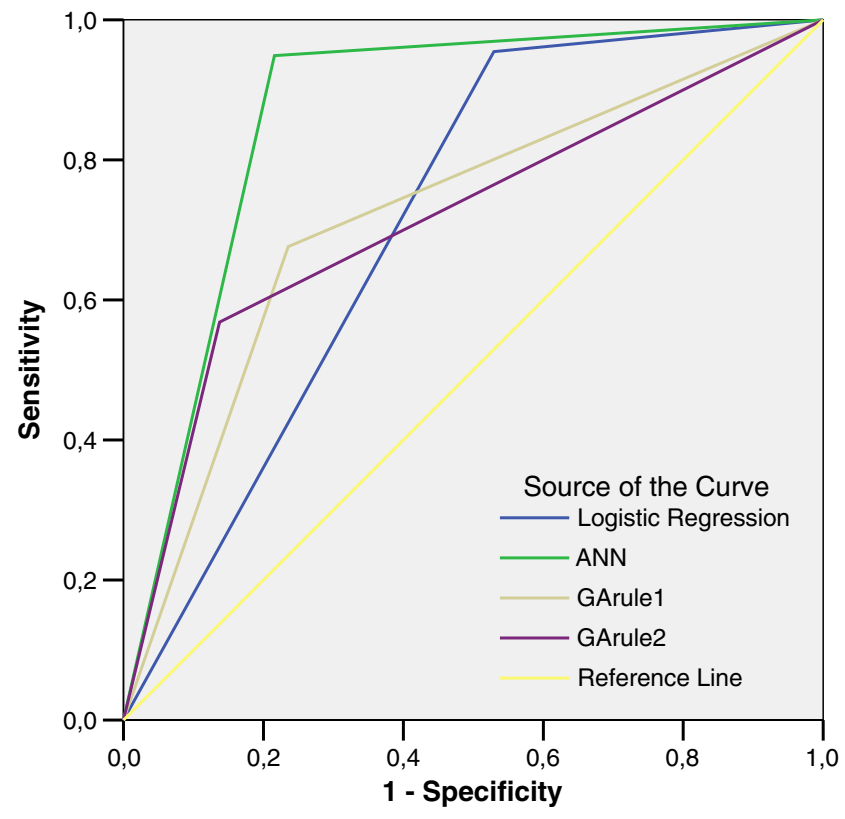

Fig. 3 ROC analyses comparing all the applications in predicting urinary stones as binary outcome 
Acknowledgement This study was supported by Akdeniz University Investigation Foundation. We are also grateful to the residents and physicians in the emergency department.

\section{References}

1. Hodgkin AL, Huxley AF (1952) A quantitative description of membrane current and its application to conduction and excitation in nerve. J Physiol 117:500-544

2. McCulloch WS, Pitts WA (1943) A logical calculus of ideas immanent in nervous activity. Bull Math Biophys 5:115-133

3. Hubel DH, Wiesel TN (1962) Receptive fields, binocular interaction and functional architecture in the cat's visual cortex. J Physiol 160:106-154

4. Holland J (1975) Adaptation in natural and artificial systems. University of Michigan Press, Ann Arbor

5. Goldberg DE (1989) Genetic algorithms in search, optimization, and machine learning. Addison-Wesley, Reading

6. Kartal M, Eray O, Erdogru T, Yilmaz S (2006) Prospective validation of a current algorithm including bedside US performed by emergency physicians for patients with acute flank pain suspected for renal colic. Emerg Med J 23:341-344

7. Chiang D, Chiang HC, Chen WC, Tsai FJ (2003) Prediction of stone disease by discriminant analysis and artificial neural networks in genetic polymorphisms: a new method. BJU Int 91:661-666

8. Kuzmanovski I, Zografski Z, Trpkovska M et al (2001) Simultaneous determination of composition of human urinary calculi by use of artificial neural networks. Fresenius J Anal Chem 370:919-923

9. Cummings JM, Boullier JA, Izenberg SD et al (2000) Prediction of spontaneous ureteral calculous passage by an artificial neural network. J Urol 164:326-328
10. Poulakis V, Dahm P, Witzsch U et al (2003) Prediction of lower pole stone clearance after shock wave lithotripsy using an artificial neural network. J Urol 169:1250-1256

11. Tanthanuch M, Tanthanuch S (2004) Prediction of upper urinary tract calculi using an artificial neural network. J Med Assoc Thai $87: 515-518$

12. Harrison RF, Kennedy RL (2005) Artificial neural network models for prediction of acute coronary syndromes using clinical data from the time of presentation. Ann Emerg Med 46(5):431439

13. Jaimes F, Farbiarz J, Alvarez D, Martínez C (2005) Comparison between logistic regression and neural networks to predict death in patients with suspected sepsis in the emergency room. Crit Care 9(2):R150-R156 Epub 2005 Feb 17

14. Heckerling PS, Gerber BS, Tape TG, Wigton RS (2003) Prediction of community-acquired pneumonia using artificial neural networks. Med Decis Making 23(2):112-121

15. Bektaș F, Eken C, Soyuncu S, Kilicaslan I, Cete Y (2008) Artificial neural network in predicting craniocervical junction injury: an alternative approach to trauma patients. Eur J Emerg Med 15(6):318-323

16. Bibi H, Nutman A, Shoseyov D, Shalom M, Peled R, Kivity S, Nutman J (2002) Prediction of emergency department visits for respiratory symptoms using an artificial neural network. Chest 122(5):1627-1632

Cenker Eken is 34 years old and has been working in the Emergency Department of Akdeniz University Medical Faculty, in Turkey, as an assistant professor. He started his residency program in 2000 and finished it in 2004. He is particularly interested in research, biostatistics, and evidence-based medicine. 